\title{
Modeling the Dynamics of Recognition-Primed Decision Making
}

\author{
Robert Patterson \\ Washington State University \\ Link Simulation and Training \\ rpatter@mail.wsu.edu
}

\section{Byron Pierce}

Air Force Research Laboratory

byron.pierce@mesa.afmc.af.mil

\author{
Lisa Fournier \\ Washington State University \\ lfournier@wsu.edu
}

\author{
Marc Winterbottom \\ Air Force Research Laboratory \\ marc.winterbottom@mesa.afmc.af.mil
}

\author{
Lisa Tripp \\ Washington State University \\ lismarie41583@yahoo.com
}

\begin{abstract}
Motivation-Two decision-making processes have been identified: an analytical process and an intuitive process. One conceptual model of the latter is the Recognition Primed Decision (RPD) model (Klein, 2008). According to this model, decision making in naturalistic contexts entails a situational patternrecognition process which, if subsequent expectancies are confirmed, lead the decision maker to render a decision to engage in a given course of action. Research approach-In this paper, we describe a system dynamics model of Klein's RPD framework, focusing upon the dynamics of the decision process. Findings/Design-Our framework is based on a model of a set of laboratory phenomena called conjunction benefits and conjunction costs, which was extended to encompass the RPD framework. Research limitations/Implications-Our simulations suggest that decision priming (bias in decision making) should occur in many naturalistic settings. Originality/Value-Originality comes from system dynamics modeling. Take away message-Robust decision making based on pattern recognition may be susceptible to priming.
\end{abstract}

Keywords

Recognition-primed decision model, intuitive decision making, system dynamics modeling

\section{INTRODUCTION}

Many authors (e.g., Evans, 2008; Sloman, 1996) have proposed that human reasoning is composed of a blend of two systems, an analytical system and an intuitive system. Analytical reasoning refers to the rendering of conscious decisions that entail the contrasting of options. Intuitive reasoning (knowing without deliberation) refers to the rendering of decisions whose steps may be unconscious and is based on situational pattern recognition.

\section{Recognition-Primed Decision Making}

Klein $(1997,2008)$ proposed a conceptual model of intuitive pattern-recognition-based decision making called the recognition-primed decision ( $R P D)$ model, which is composed of three components: a matching component, a diagnose component, and a simulate-course-of-action component. Figure 1 depicts the matching component and the diagnose component; the former is represented by the box labeled "pattern recognition" and the latter by the box labeled "clarify/diagnose (story building)."

In this model, an individual with expertise identifies a problem situation as typical and familiar, based upon a match to an analog situation stored in memory, and as expectations become confirmed over time, he or she decides to initiate an action. If the situation is unfamiliar, or if subsequent expectations are violated, then the individual decides to diagnose the situation further before acting, which may include story building. An individual may also mentally evaluate and simulate a course of action before implementing it; however, in this paper we do not concern ourselves with this component. In this paper, we present a formal computational model of the dynamics of Klein's RPD model employing system dynamics methodology. 


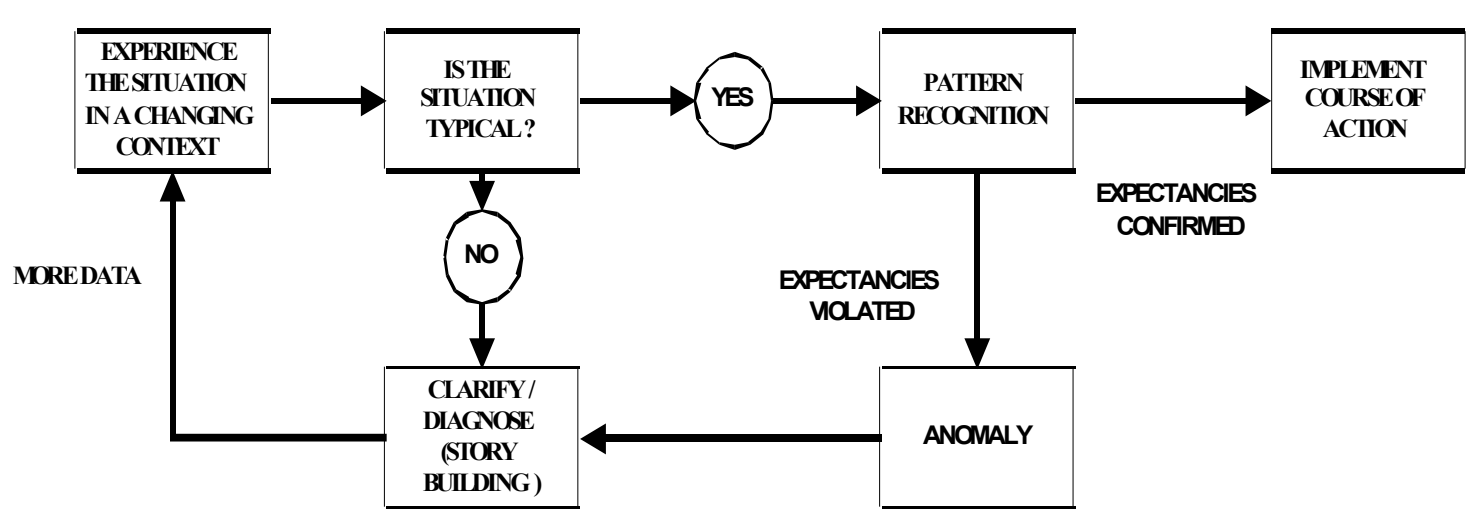

Figure 1. Diagram of the Recognition-Primed Decision model (adapted from Klein, 1997, 2008).

We focus on the match and diagnose components of Klein's model, which convey the idea that an initial pattern (situation) matching process is first undertaken, followed by a subsequent situational assessment that entails a further pattern matching process that may confirm or violate expectations. If both the initial situational pattern is matched and expectations are subsequently confirmed, then a decision to act is made.

This scenario implies a framework based on parallel information-processing channels with nested decisional operators (PCNDO). One type of operator would be an OR gate, which would exist for a pair of channels signaling an initial pattern match or non-match, and a second OR gate, which would exist for another pair of channels signaling expectancies confirmed or violated. The output from the two pairs of channels would be combined with an AND gate which would lead to a final decision. An analogous type of model has been described by Townsend and Wenger (2004).

This type of system can lead to interesting phenomena, especially when dynamics are considered. Specifically, this type of system can lead to decision priming when the activation of two parallel channels is staggered in time. An example of this type of effect comes from a set of laboratory phenomena called conjunction benefits and conjunction costs (CBCC), which can also be placed in a PCNDO framework.

\section{Conjunction Benefits and Conjunction Costs}

In the CBCC research (e.g., Fournier, Eriksen \& Bowd, 1998; Fournier, Patterson, Dyre, Wiediger, \& Winters, 2007), an individual has to render a judgment about the presence or absence of a conjunction of two target features in a brieflyexposed stimulus. When two stimulus features differ in their speed of discrimination, correct decisions indicating the presence of the conjunction are faster than correct responses to the single feature for which discrimination is slowest, which indicates that the feature for which discrimination is fastest primes the decision made about conjunction. This is conjunction benefits.

Correct decisions indicating the absence of a conjunction are delayed if the feature for which discrimination is the fastest is present, relative to when neither target feature is present, which also indicates that a single feature can prime decisions made about the conjunction. It is assumed that the target feature for which discrimination is the fastest incorrectly primes a central decision process that the target conjunction is present. Delayed activation resulting from the feature for which discrimination is the slowest must override this primed decision, which takes additional processing time. This is conjunction costs.

Representative data from Fournier et al. (2007) show the following: reaction time (RT) for rendering a correct "present" decision about the fastest feature by itself was $460 \mathrm{msec}$, whereas it was $525 \mathrm{msec}$ for the slowest feature. RT for rendering a correct "present" decision about the conjunction of the two features was $490 \mathrm{msec}$, which was faster than the RT for the slowest feature (conjunction benefits). RT for rendering a correct "absent" decision about the conjunction was $620 \mathrm{msec}$ when the fastest target feature was present (but the slowest target feature was absent), relative to an RT of $490 \mathrm{msec}$ when neither target feature was present (conjunction costs).

In the present study, we developed a system dynamics model of the dynamics of the CBCC phenomena, and generalized that structure to Klein's RPD model. Our computational model of the CBCC phenomena was validated by comparing its output to the CBCC trends reported by Fournier et al. (2007). Our model RTs: fastest feature: $438 \mathrm{~ms}$; slowest feature: $538 \mathrm{~ms}$; conjunction present: $513 \mathrm{~ms}$. Simulated RT for the conjunction present response fell in 
between the simulated RT values for the fastest and slowest feature responses; the model simulated conjunction benefits. Our model also generated the following RTs: neither feature present (conjunction absent response): $513 \mathrm{~ms}$; fastest feature present only (also conjunction absent response): $>975 \mathrm{~ms}$. Simulated RT for the conjunction absent response when the fastest feature was present was longer than RT for the same response when neither feature was present; the model simulated conjunction costs. We next re-calibrated the CBCC model structure for use as a framework for Klein's RPD model, shown below.

There have been other models of the RPD process (Warwick, McIlwaine, Hutton \& McDermott, 2001; Mueller, 2009). While interesting, those efforts did not focus on the dynamics of decision making.

\section{System Dynamics Modeling}

System dynamics was created by Forrester (e.g., 1961) as an approach for analyzing complex systems that possess feedback processes, such as business systems, urban systems, or natural ecological systems. To do so, computational structures are created that represent systems of differential equations. The computational structure of a system is created by using a small set of elements that are interconnected in complex ways. These elements include a 'stock', which represents integration (represented in diagrams as a rectangle); a 'flow', which represents rate of change or a derivative (in diagrams, a thick arrow); a 'connector', which represents feedback and other types of connections (in diagrams, a thin arrow); and a 'converter', which represents a constant, variable, expression, or conditional logic (in diagrams, a circle). The solution to the system of differential equations can be estimated by solving a system of difference equations using stepwise numerical integration (Sterman, 2000).

\section{RPD MODEL STRUCTURE}

In our model of Klein's RPD framework, the initial situation was represented as a pair of information flows, with each flow broken into two parts: initial familiar-situation information followed by pattern recognition, or initial unfamiliarsituation information followed by pattern non-recognition. Subsequent information confirming or violating expectations was also represented as a pair of information flows, with each flow broken into two parts: subsequent other information A followed by expectancies confirmed, or subsequent other information B followed by expectancies violated. Thus, each lower-level decision concerning recognition or expectancies was represented by two flows, one for each decision state or alternative, which were combined with an OR decision operator; and each flow was represented as a discrete two-stage process (Meyer, Yantis, Osman \& Smith, 1985). Only one flow of each pair was active at any one point in time. The processing flows for the lower-level decisions (recognition and expectancies) were combined with an AND operator.

Our system dynamics RPD model is shown in Figure 2. Here, stocks (rectangles) are integrators that accumulate various levels of information, activation, or decision commitment (all stocks have a capacity of 100\%).

In Figure 2, information processing flows from the left side of the figure to the right side. The model was composed of three levels: (1) initial integration processes involving different information, shown down the left side of the figure; (2) mental events concerning recognition or expectancies, depicted down the middle of the figure; (3) a central-decision process rendering an 'implement course of action' decision, or a 'diagnose' decision, shown on the right side of the figure.

Other features: (1) decision making took 10-20 seconds, consistent with decision making by fire ground commanders (G. Klein, personal communication, August 15, 2008); (2) information accumulates in a time-variant fashion (Ditterich, 2006); (3) our model was deterministic (lacked stochasticity); (4) we did not implement the cyclic nature of Klein's model. 


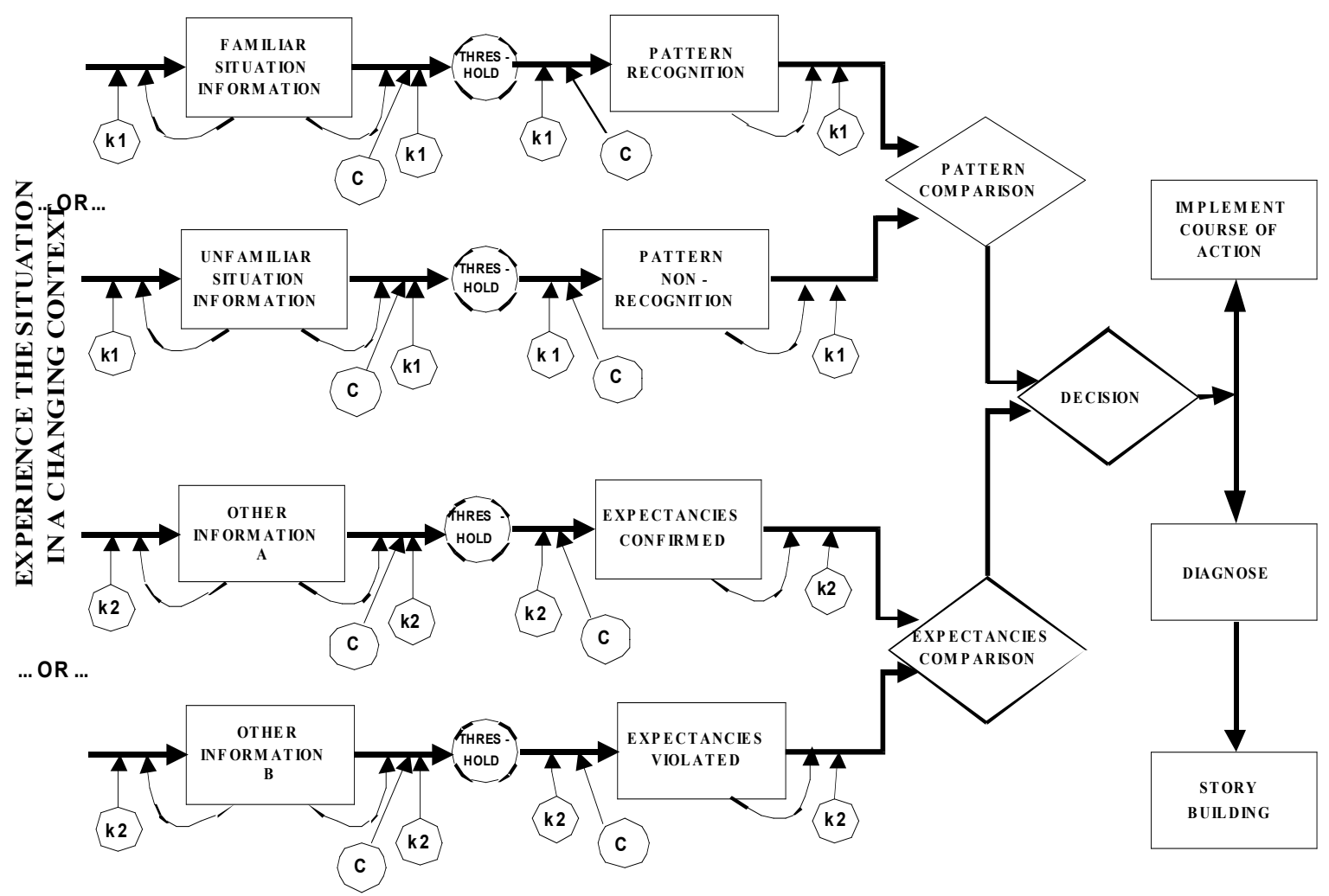

Figure 2. System dynamics model of Recognition-Primed Decision model. Pattern-comparison and expectancies-comparison processes: logical OR operators; decision process shown as a diamond: logical AND operator.

\section{Initial Integration Processes}

Initial integration processes integrated information about a given situation, which was consistent with either a familiar situation or an unfamiliar situation, and supplemented with subsequent information that either confirmed expectancies ('other information A') or violated expectancies ('other information B'). Integration processes were capacitated growth processes based on a logistic function. The expression for these integration processes was (using familiar-situation information as an example):

$$
\text { net growth rate of information }=\frac{d F S I}{d t}=(k \cdot F S I)-\left(k \cdot F S I \cdot \frac{F S I}{C}\right)
$$

where FSI = familiar situation information, $t=$ time, $k=$ growth/decay fraction $(k=k 1$ or $k 2)$, and $C=$ capacity. Here, $k 1>k 2$, thus situational information accumulated at a faster rate than other information. The model represents a given decision-making scenario by activating a given combination of initial integration processes with a starting value of 10 (e.g., $\operatorname{FSI}(0)=10)$. To de-activate, initial value was 0 .

A threshold mechanism whose value was 50 (half the perceptual information was to be accumulated before subsequent processing) followed each initial integration mechanism, creating a time delay:

$$
\text { IF FSI > 50, THEN FSI. ELSE } 0 .
$$

\section{Recognition/Expectancy Mechanisms}

The mechanisms for pattern recognition and expectation integrated the information from the initial integration processes to generate a time-limited response: a recognition or non-recognition response, and an expectancies-confirmed or 
expectancies-violated response. The response of these mechanisms was a rounded pulse profile which decayed to zero when the corresponding prior initial integration process (equation 1) reached full capacity. Net growth rate of these mechanisms was, using the pattern recognition mechanism as example:

$$
\frac{d P R}{d t}=\left[(k \cdot F S I) \cdot\left(1-\frac{F S I}{C}\right)\right]-(k \cdot P R),
$$

where FSI, $k, t$ and $C$ were as defined above and $P R$ was the level of the pattern recognition response. This produced an approximately bell-shaped pulse in its output (provided $k$ was not zero).

Output from the recognition and expectation mechanisms projected to processes called 'pattern comparison' and 'expectancies comparison', each of which compared one type of response to determine whether the information was consistent with an implement-course-of-action decision:

IF (Pattern Recognition > Pattern Nonrecognition) THEN (Pattern Recognition * $\beta$ )

ELSE (Pattern Nonrecognition *- $\beta$ ),

where $\beta$ is a scaling factor that kept information emanating from the pattern-comparison process and the expectanciescomparison process balanced. The output from these two comparison processes projected to the central decision process.

\section{Central Decision Process}

The central decision process rendered a final decision to either implement a course of action, or to diagnose the situation further (leading to a story-building algorithm). The central decision process integrated information from the two comparison mechanisms and determined whether both a familiar situation was perceived and other information confirmed expectations. The central decision process was composed of two interconnected decision reservoirs, and was activated by a logic statement (called 'Decision' in the diamond in Figure 2) which compared the streams of information coming from the pattern-comparison process and expectancies-comparison process:

IF $[($ Pattern Comparison $>0)$ AND $($ Expectancies Comparison $<0)]$ OR $[($ Expectancies Comparison $>0)$ AND $($ Pattern Comparison < 0)] THEN (MAX (Pattern Comparison, Expectancies Comparison) *-1) + MIN (Pattern Comparison, Expectancies Comparison) ELSE (Pattern Comparison + Expectancies Comparison).

Each decision reservoir symbolized the amount of decision commitment for each decision: 'implement course of action' or 'diagnose the situation'. The simulation began with decision ambiguity (50 in each of the two reservoirs). Over time, amount of decision commitment flowed from one reservoir and into the other, depending on incoming evidence from pattern and expectancies comparison processes. Rate of flow into or out of one or the other reservoir was determined by the sum of the activation levels of the comparison processes; for the "implement course of action" reservoir:

$$
I C A=\int C D G R d t+I C A(0)
$$

where $I C A=$ level of commitment to the implement-course-of-action decision, $C D G R$ (central decision growth rate) $=$ Pattern-Comparison + Expectancies-Comparison, and $I C A(0)$ is the initial value of $I C A$, where $I C A(0)=50$. An analogous definition would apply to "diagnose" reservoir.

\section{MODELING RESULTS}

The model was implemented using the Stella software package (ISEE Systems, Inc., Waltham, MA). The Euler method of numerical integration was used with $\mathrm{dt}=0.1$. For the pattern-comparison process, $k 1=1.0$ and $\beta=+/-0.65$; for the expectancies-comparison process, $k 2=0.4$ and $\beta=+/-0.65$. 

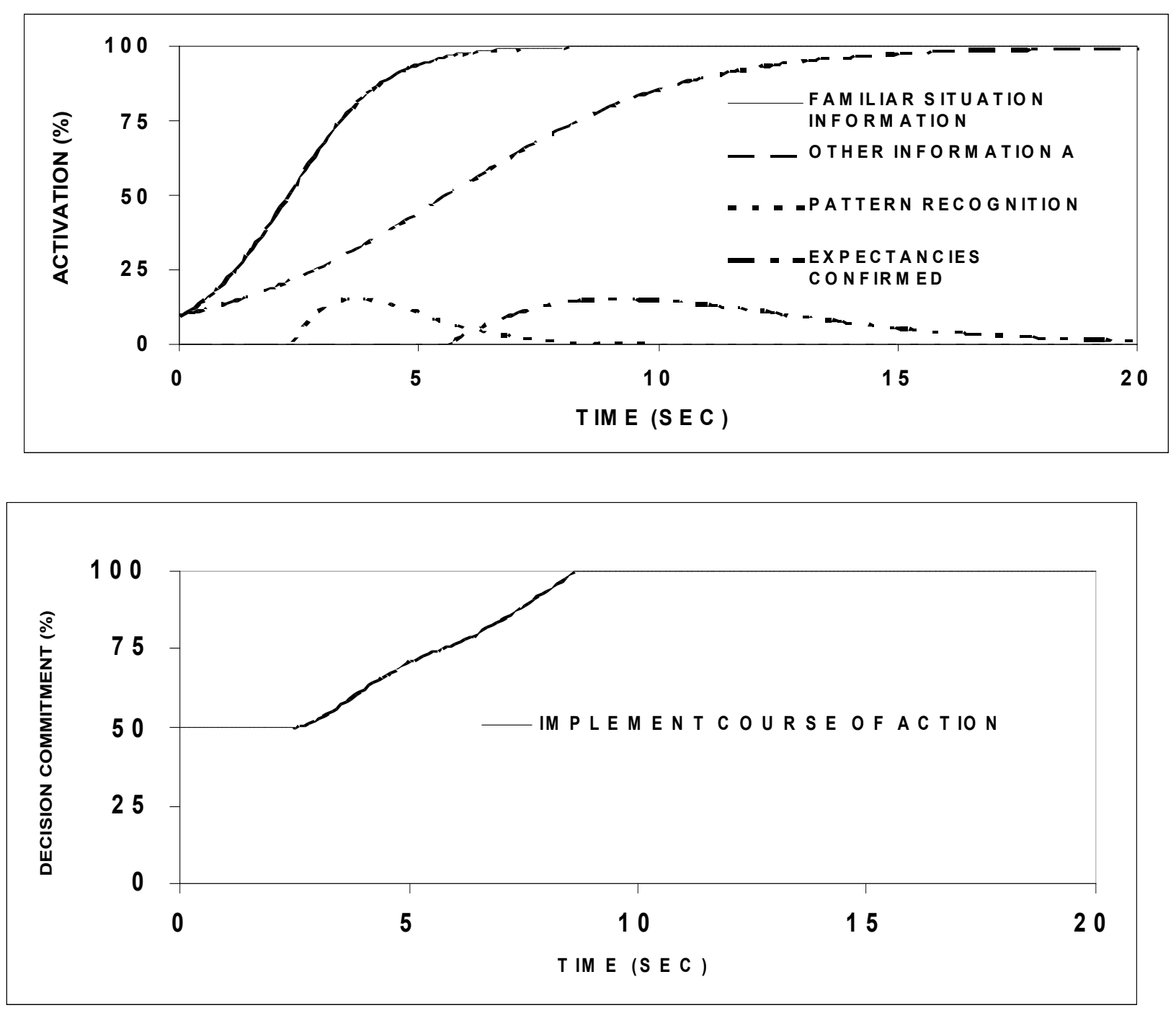

Figure 3. Typical/familiar situation with expectancies confirmed.

Figure 3 shows simulation of the "implement course of action" decision. Solid and dotted curves, upper panel, show the integration of the familiar-situation information, and the response of the pattern recognition mechanism, respectively. Dash and dash-dot-dash curves show the integration of other information A, and the response of the expectanciesconfirmed mechanism, respectively. Final decision to implement a course of action ( $100 \%$ decision commitment) took 8 seconds (Figure 3, lower panel). 


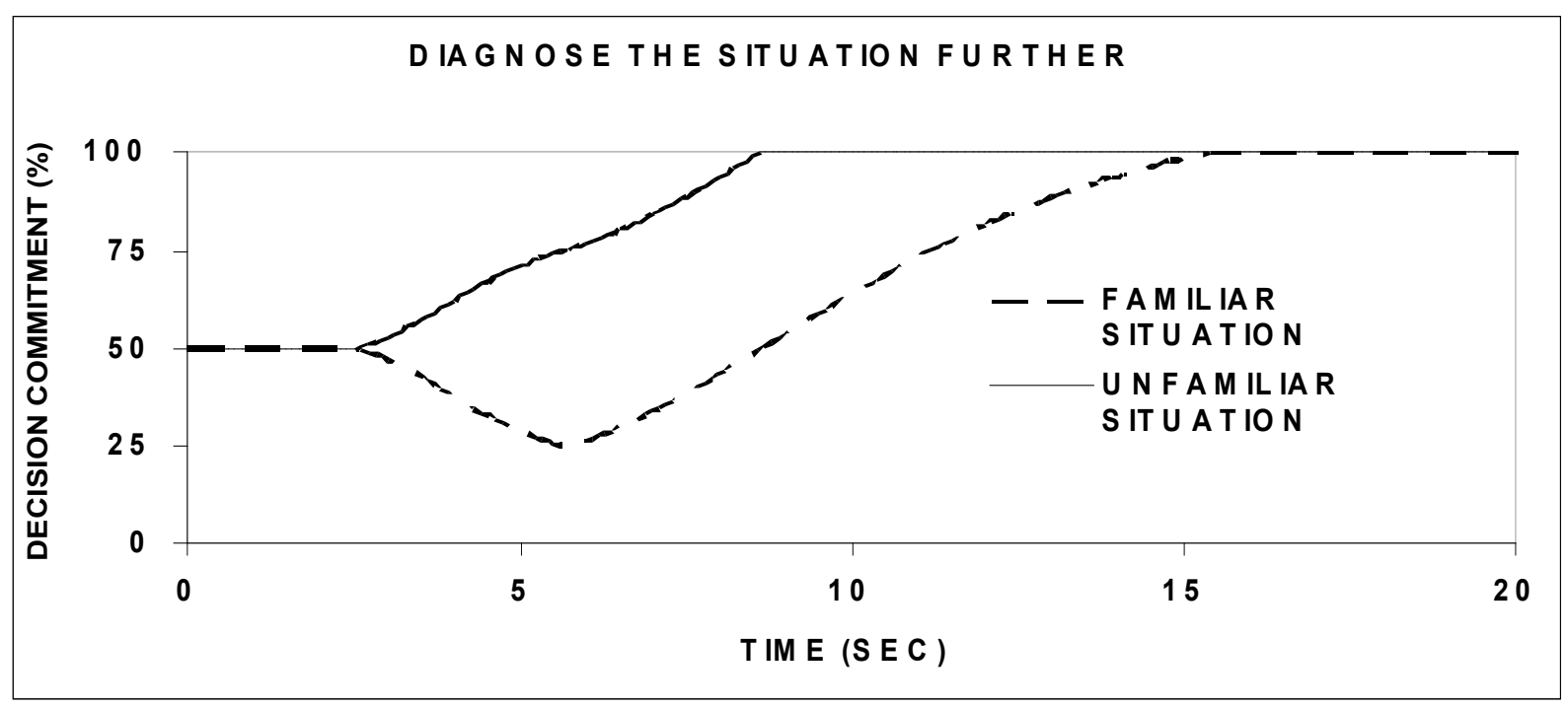

Figure 4. Time course of commitment to the decision to diagnose the situation further.

Figure 4 shows simulation of the "diagnose the situation further" decision under two conditions: (1) non-recognition of an unfamiliar situation and violated expectancies, which took 8 seconds; and (2) recognition of an initial familiar situation but subsequent violated expectancies, which took 16 seconds. For the latter, initial familiar situational information primed the central decision process to begin to decide to "implement a course of action", but subsequent information produced an expectancies-violated response, and the central decision process had to reverse itself to produce a 'diagnose' decision.

Once the decision was made to diagnose the situation further, the process of story building was undertaken, which took about 60 seconds to complete. Story building was modeled as a logistic function, with $k=0.15$ (figure not shown).

Overall, the model takes about 8 seconds to decide to implement a course of action, or between 8-16 seconds to decide to diagnose a situation further, depending upon the presence or absence of conflicting information. It takes an additional 60 seconds to perform a story building operation following the decision for diagnosis (Figure 5).

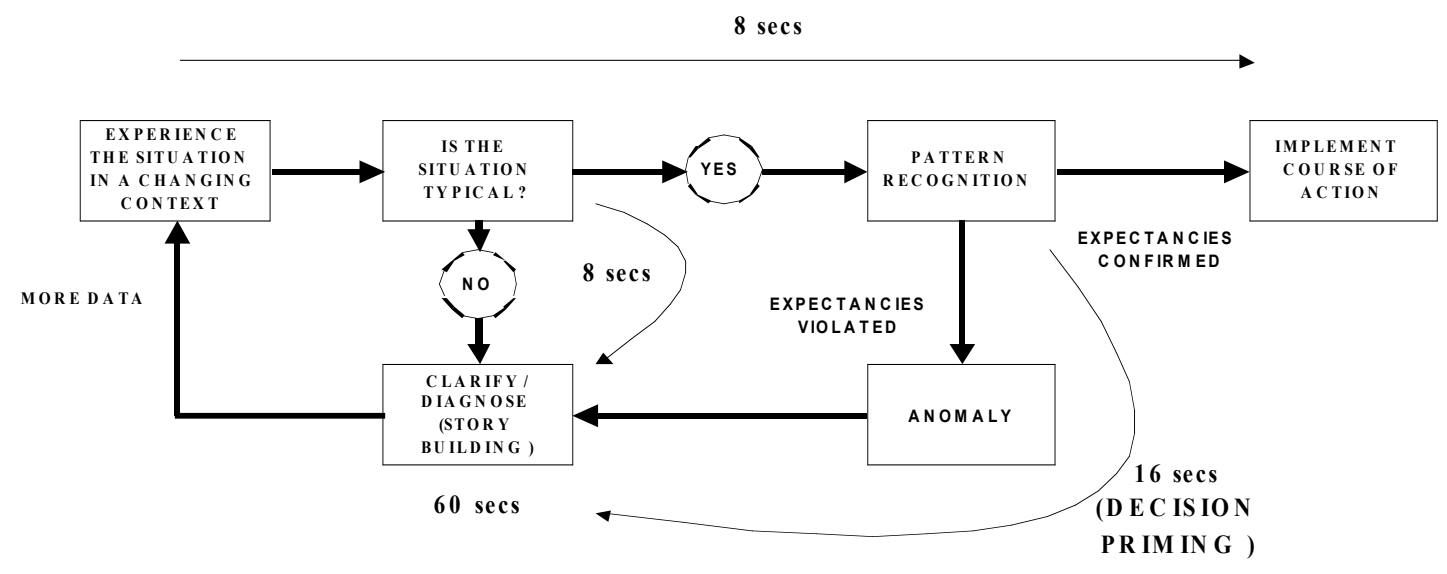

Figure 5. Diagram of the Recognition-Primed Decision model (Klein, 1997, 2008) with simulated latencies.

Our model does not depend upon the timing shown in Figure 6, and an analogous pattern of results would occur with changes in temporal scale so long as the relative timing of the multiple events is sufficiently lagged. We predict that the time it takes to make pattern-based decisions depends on whether incoming information is compatible or conflicting. 


\section{CONCLUDING REMARKS}

The validity of our model can be tested by empirically determining whether decision priming actually occurs in naturalistic contexts. Mitchell and Flin (2007) recently reported that they failed to find evidence for decision priming when police officers were responding to simulated threats in a firearms training simulator. In an attempt to induce decision priming, these authors had the officers hear a threat or neutral briefing information prior to experiencing shoot or no-shoot scenarios. The results showed that the type of briefing had no effect on the officers' decision to shoot.

One reason for the failure to observe decision priming in the Mitchell and Flin study may have been that the decisionmaking process would have been inherently intuitive and driven by situational pattern recognition, yet the briefing information was given verbally. Because verbal material is related to the analytical reasoning process (Evans, 2008; Sloman, 1996), it may have had less of an impact on intutive decision making than had these authors used a perceptual/situational priming stimulus. Our model of Klein's RPD framework, and the model of the CBCC phenomena upon which it was based, involved perceptual/situational priming stimuli.

If validated, and with further elaboration, our model might potentially be used in the development of decision support tools and training aids. For example, increasing the complexity of the model to deal with multiple priming stimuli may help predict conditions under which priming would be enhanced versus conditions under which it would be eliminated. This information could help in the design of immersive decision environments by creating perceptual cues that are selectively presented for priming decision making when the individual faces information overload.

\section{ACKNOWLEDGMENTS}

The authors thank Dr. Shane T. Mueller, Klein Associates Division, ARA, Inc., for very helpful comments on a previous version of this manuscript. This work was supported by U.S. Air Force contract FA8650-05-D6502 to Link Simulation and Training (a division of L3 Communications Corp.). The views expressed are those of the authors; they do not reflect the official policy or position of the Department of Defense or U.S. Government. This paper was cleared for public release.

\section{REFERENCES}

Ditterich, J. (2006). Evidence for time-variant decision making. European Journal of Neuroscience, 24, 3628-3641.

Evans, J.S.B.T. (2008). Dual-processing accounts of reasoning, judgment and social cognition. Annual Review of Psychology, 59, 255-278.

Forrester, J.W. (1961). Industrial dynamics. Waltham, MA: Pegasus Communications.

Fournier, L. R., \& Eriksen, C. W., \& Bowd, C. (1998). Multiple feature discrimination faster than single feature discrimination within the same object? Perception \& Psychophysics, 60, 1384-1405.

Fournier, L., Patterson, R., Dyre, B.P., Wiediger, M. \& Winters, R. (2007). Conjunction benefits and costs reveal decision priming for first-order and second-order features. Perception \& Psychophysics, 69, 1409-1421.

Klein, G. (2008). Naturalistic decision making. Human Factors, 50, 456-460.

Klein, G. (1997). The recognition-primed decision (RPD) model: Looking back, looking forward. In C.E. Zsambok \& G. Klein (Eds.), Naturalistic Decision Making. Mahwah, N.J.: Lawrence Erlbaum Ass.

Mueller, S.T. (2009). A Bayesian recognitional decision model. Journal of Cognitive Engineering and Decision Making, in press.

Sloman, S.A. (1996). The empirical case for two systems of reasoning. Psychological Bulletin, 119, 3-22.

Sterman, J.D. (2000). Business dynamics: Systems thinking and modeling for a complex world. Boston, MA: McGrawHill.

Townsend, J.T. \& Wenger, M.J. (2004). A theory of interactive parallel processing: New capacity measures and predicitons for a response time inequality series. Psychological Review, 111, 1003-1035.

Warwick, W., McIlwaine, S., Hutton, R. J. B., \& McDermott, P. (2001). Developing computational models of recognition-primed decision making. Proceedings of the Tenth Conference on Computer Generated Forces, Norfolk, VA. SISO. 